\title{
From Traditional to Automated Mobility on Demand: A Comprehensive Framework for Modeling On-Demand Services in SimMobility
}

Nahmias-Biran, Bat Hen; Oke, Jimi B.; Kumar, Nishant; Basak, Kakali; Araldo, Andrea; Seshadri, Ravi; Akkinepally, Arun; Lima Azevedo, Carlos M.; Ben-Akiva, Moshe

\section{Published in:}

Transportation Research Record

Link to article, DOI:

$10.1177 / 0361198119853553$

Publication date:

2019

Document Version

Peer reviewed version

Link back to DTU Orbit

Citation (APA):

Nahmias-Biran, B. H., Oke, J. B., Kumar, N., Basak, K., Araldo, A., Seshadri, R., Akkinepally, A., Lima Azevedo, C. M., \& Ben-Akiva, M. (2019). From Traditional to Automated Mobility on Demand: A Comprehensive Framework for Modeling On-Demand Services in SimMobility. Transportation Research Record, 2673(12). https://doi.org/10.1177/0361198119853553

\section{General rights}

Copyright and moral rights for the publications made accessible in the public portal are retained by the authors and/or other copyright owners and it is a condition of accessing publications that users recognise and abide by the legal requirements associated with these rights.

- Users may download and print one copy of any publication from the public portal for the purpose of private study or research.

- You may not further distribute the material or use it for any profit-making activity or commercial gain

- You may freely distribute the URL identifying the publication in the public portal 


\section{FROM TRADITIONAL TO AUTOMATED MOBILITY ON DEMAND: A COMPREHENSIVE FRAMEWORK FOR MODELLING ON DEMAND SERVICES IN SIMMOBILITY}

Bat Hen Nahmias-Biran ${ }^{1,2}$, Corresponding Author

E-mail: bathennb@ariel.ac.il; $\underline{\text { bathen@ mit.edu }}$

Jimi B. Oke ${ }^{3}$

E-mail: oke@mit.edu

Nishant Kumar ${ }^{2}$

E-mail: nishant@smart.mit.edu

Kakali Basak ${ }^{2}$

E-mail: $\underline{\text { kakali@smart.mit.edu }}$

Andrea Araldo ${ }^{4,3}$

E-mail: araldo@mit.edu

Ravi Seshadri ${ }^{2}$

E-mail: $\underline{\text { ravi@smart.mit.edu }}$

Arun Akkinepally ${ }^{3}$

E-mail: arunprak@mit.edu

Carlos Lima Azevedo 5

E-mail: climaz@dtu.dk

Moshe Ben-Akiva ${ }^{2,3}$

30 E-mail: $\underline{\text { mba@mit.edu }}$

${ }^{1}$ Department of Civil Engineering, Faculty of Engineering, Ariel University, Ariel 40700, Israel

${ }^{2}$ Singapore-MIT Alliance for Research and Technology, 1 Create Way, 138602, Singapore

${ }^{3}$ Intelligent Transportation Systems Lab, Massachusetts Institute of Technology, Cambridge, MA 02139

${ }^{4}$ SAMOVAR, CNRS, Télécom SudParis, Institut Polytechnique de Paris

${ }^{5}$ Department of Management Engineering, Technical University of Denmark, 2800 Kgs. Lyngby, Denmark

45 Word count: $\mathbf{7 0 0 0}$ words text $+\mathbf{2}$ tables $\times 250$ words $($ each $)=\mathbf{7 5 0 0}$ words

46 Submission Date: April 16, 2019 


\section{ABSTRACT}

2 Mobility-on-demand (MoD) systems have recently emerged as a promising paradigm for

3 sustainable personal urban mobility in cities. In the context of multi-agent simulation technology,

4 the state-of-the-art lacks a platform that captures the dynamics between decentralized driver's

5 decision-making and the centralized coordinated decision making. This work aims to fill this gap

6 by introducing a comprehensive framework that models various facets of MoD, namely

7 heterogenous MoD driver's decision making and coordinated fleet management within

8 SimMobility, an agent- and activity-based demand model integrated with a dynamic multi-modal

9 network assignment model. To facilitate such a study we propose an event-based modelling

10 framework. Behavioral models were estimated to characterize decision making of drivers using a

11 GPS dataset from a major MoD fleet operator in Singapore was used. The proposed framework

12 was designed to accommodate behaviors of multiple on-demand services such as traditional

13 MoD, Lyft-like services and Automated MoD (AMoD) services which interact with traffic

14 simulator and a multi-modal transportation network. We demonstrate the benefits of the

15 proposed framework through a large-scale case study in Singapore comparing the fully

16 decentralized traditional MoD with the future AMoD services in a realistic simulation setting.

17 We found that AMoD results in more efficient service even with increased demand. Parking

18 strategies and fleet size will also have an effect on user satisfaction and network performance. 


\section{INTRODUCTION}

2 A majority of past research efforts have been devoted to modelling and optimizing Mobility on 3 Demand (MoD) fleet operations (1). Much less attention has been made to the decentralized nature of MoD decision making process (2). Such nature arises from the dependency of current MoD systems on drivers and their decision power. In the context of multi-agent simulation technology, although some facets of centralized MoD operations — such as street pick-ups, queueing, routing, and fleet dispatch — have been modelled, there is no platform that captures the dynamics between decentralized driver's decision-making and the centralized decision making. Such decentralized perspective is critical in modeling MoD systems and the potential impacts of automation, as drivers can only be informed, incentivized or coordinated but not centrally controlled (3).

This work aims to fill this gap by introducing a comprehensive framework that models various facets of MoD driver's behavior along with a decentralized fleet management system within an agent-based demand-supply simulator, SimMobility Mid-Term. SimMobility Mid-Term (MT) simulator is an agent- and activity-based demand model integrated with a dynamic multimodal network assignment model (4). The traffic dynamics are simulated using a multi-modal mesoscopic simulator (supply simulator). MT is part of a much larger simulation platform that also contains long term and short-term models. Simulating MoD services is extremely challenging because of complex interactions between independent drivers, the central controller, and travelers' decision processes. To facilitate the study of such a complex and partially decentralized system, we propose an event-based modelling framework. In this framework, the drivers, the controllers, and the travelers are represented as separate decision agents making plans and event-triggered actions. Behavioral models were estimated to characterize decision making of drivers using a GPS dataset from a major MoD fleet operator in Singapore in 2013, containing position and service status data over 30 days. A unified framework was developed to model the operation of both traditional MoD fleets and emerging ride-hailing services such as Uber, and Lyft-like services. The specific behaviors of MoD service drivers are modelled within a discrete-choice framework. Specifically, the following models are proposed: (i) Break, (ii) Cruise/ Not to Cruise, (iii) Stand Choice (choice among the available MoD stand), (iv) Zone Choice during cruising Model and (v) Route Choice. The suggested models can reflect strategic decisions made by the driver.

While traditional MoD actions are made by the driver, some MoD services can coordinate some of the processes above. Ultimately, automated MoD (AMoD) services could fully control and optimize all decisions making processes. The AMoD controller would for example process traveler's service requests and assign them to a given vehicle after considering its current occupancy (and potential route), whether the passenger is willing to share the ride or not, time to reach to the pick-up location and the travel time to final destination. Thus, an MoD controller agent in simulation should capture MoD service status, updated vehicle locations and monitor vehicle movement through the network, reacting to incoming requests and changes on network and fleet performance accordingly. A framework to handle MoD controllers in SimMobility was presented in (4) and integrated within the calibrated SimMobility model of Singapore [for details on the estimation, implementation and validation of the Singapore model, the reader is referred to (4). In this paper, we have extended the MoD controller framework with several features for service driver behavior modelling and simulation, and demonstrate it through a case study of 
Singapore, where different MoD services are been modeled. Specifically, traditional MoD is simulated and compared to AMoD. The current work has five major contributions: (1) development of a comprehensive event-based framework that addresses complex behaviors and interactions of service drivers, $\mathrm{MoD}$ centralized operation, and travelers; (2) incorporation of the proposed framework within a highly realistic agent-based simulation platform, SimMobility; (3) evaluation of the suggested framework against real-world data; (4) demonstration of the proposed framework through a case study of Singapore; and (5) showcase the use of the proposed framework in the evaluation of potential mechanisms and policies when deploying MoD services.

The rest of the paper is structured as follows. Section 2 provides a literature review on recent $\mathrm{MoD}$ and MoD services modeling and simulation. Section 3 introduces the MoD framework, including the behavioral models and the MoD controller. Section 4 includes a case study demonstrating the use of the $\mathrm{MoD}$ framework for modeling traditional MoD vs $\mathrm{AMoD}$ services in Singapore. Finally, Section 5 presents the main conclusions and findings of this work.

\section{LITERATURE REVIEW}

Many past research efforts have been devoted to the modeling of the MoD fleet operations. Leveraging on the current extensive review of MoD service modeling and simulation literature (1), we discuss the latest studies, focusing on three streams: (1) large scale simulations of MoD services; (2) theoretical and mathematical models to describe different MoD services aspects, and (3) data driven studies of MoD behavior.

Within the context of large-scale simulation of MoD services, majority of past research used microscopic simulation. (6) used MATSim for the modeling of MoD services in Barcelona and Berlin and focused on assessing the performance of two MoD dispatching strategies in balancing supply and demand. The first strategy always serves awaiting requests by dispatching the nearest idle $\mathrm{MoD}$, but this method has poor performance under high demand. The second strategy is a balancing strategy that minimizes pickup trip times instead of serving requests in the FIFO order. However, neither strategy simulated real MoD behavior. (7) also used MATSim to simulate the interaction between newly introduced autonomous vehicle MoD services with the existing means of transport. They used a simplified version of a greedy controller in a MATSim scenario of the city of Sioux Falls, South Dakota, USA. Both efforts using MATSim did not model the behavior of the MoD driver but used random choices. (8) studied booking strategies for a MoD dispatching system. The study identifies two types of booking: Current Booking (CBK), where the customers makes a booking call for a MoD to arrive as soon as possible, and Advance Booking $(\mathrm{ABK})$, where customers indicate a pickup time which is at least in half an hour later. In the simulation model, the central region of Singapore was chosen as the study area. The results of the study show that advanced booking benefits small MoD operators with comparatively low booking demands but is ineffective for larger MoD operators with high booking demands. (2) used MoDSim to model MoD behavior at the macro-level. MoDSim is designed to be a decentralized discrete event simulation; it models MoD drivers' cruising/roaming behavior while treating the traffic condition in the network as exogenous. Singapore was used as the study area. (9) developed a mathematical model for real-time high-capacity ride-sharing. The model was experimentally validated with New York City MoD data, and results showed that 98\% of MoD 
rides currently served by over 13,000 MoD drivers could be served with just 3,000 MoD vehicles via automation and sharing.

The second stream of research focuses on small scale optimization problems and empirical models used to describe the different aspects of MoD services. (10) studied the MoD dispatching system in Singapore and proposed a method where the MoD assigned a booking job is the one with the shortest time path, reaching the customer in the shortest time determined by real-time traffic conditions. The microscopic simulation was performed in a small toy network. (1) proposed and tested an agent-based simulation model focused on a shared-MoD service. The proposed system optimizes fares and travel time savings for passengers. The simulation did not include a dynamic traffic model or a dynamic demand model. (11) focused on e-hailing, proposing a spatial equilibrium model to balance supply and demand of MoD services.

(12) explored the market demand potential of a Shared-Ride MoD. They presented an integrated choice and latent variable modeling framework for modeling the number of times per week a Shared-Ride MoD would be used if it were implemented at the American University of Beirut campus. A series of studies by Wong et al. (see for example 13) extensively details the customersearching behavior of MoD drivers over different periods of time. (14) explored methods of optimizing mobility on demand personalized services. However, this simulation ignores actual traffic conditions on the network.

The third stream of research consists of data driven studies that were used to draw insight into the behavior of MoD drivers. (15) used a stated preference survey of $400 \mathrm{MoD}$ drivers conducted in 2000 in Hong Kong to estimate a multinomial logit choice model for MoD customer-searching behavior and discovered that the journey time, toll, and waiting time were found to be significant factors in the choices drivers made at the $1 \%$ level. (16) used the complete trace information from $3590 \mathrm{MoD}$ drivers in Beijing to understand passenger denial behavior of MoD drivers. (17) focused on the willingness of MoD drivers to drive to the airport empty and used AVL data from $8,954 \mathrm{MoD}$ drivers during a period of five weekdays in Shanghai. Their analysis revealed that airport-serving MoD drivers earn significantly less in most time periods during the day, but vacant-MoD drivers are still more likely to serve the airport if they have relatively higher profits in airport-originated trips. (18) investigated the factors contributing to single-trip MoD efficiency. By evaluating MoD performance using GPS data from $2000 \mathrm{MoD}$ drivers in Wuhan in 2013, they found that high-performing, efficient drivers operate further away from downtown areas and navigate through the whole city, changing locations consistently to obtain the best traffic conditions.

Methodologies used so far in the literature focused mainly on MoD dispatching algorithms with very limited large-scale applications. Others used a real-world data to understand a specific $\mathrm{MoD}$ driver behavior, but none of them tried to capture the full set of behavior of the MoD driver, or combine all the pieces into one comprehensive framework that can address the complex behaviors and interactions of $\mathrm{MoD}$ drivers, fleet controllers, travelers, congestion and other modes. In this study we intend to fill this gap by developing a comprehensive tool to predict and evaluate the impact of the transformation of on demand services using SimMobility simulator. 


\subsection{Overview of SimMobility Mid-Term Simulator}

SimMobility (MT) simulator is an agent-based, fully econometric, and activity-based demand model integrated with a dynamic traffic assignment model (3), (4). It is capable of simulating daily travel at the individual levels. The traffic dynamics are simulated using a mesoscopic simulator. Figure 1 presents the modeling framework structure of the MT simulator in SimMobility. In this specific study, at the Pre-day level (agent planning stage), different MoD services are introduced, such as traditional MoD, Uber and Lyft-like services, and possibly, AMoD services alongside with traditional modes (car, etc.) to allow the synthetic population of agents to choose from all modes of interest for the trips associated with all planned activities. These modes are included in the combined mode-destination choice models as part of an agent's choice set. The outcome of pre-day models is the Daily Activity Schedule (DAS) which is an input to the Within-day and Supply simulators. At the Within-day and Supply level, the DAS of all individuals are simulated, i.e. agent's plans become actions, and it is where the MoD Driver Behavior Framework was implemented. The MoD Driver Behavior Framework is the key innovation of this work and will be discussed in detail in the next section. Uber and Lyft-like services, and $\mathrm{AMoD}$ services are been handled by the $\mathrm{MoD}$ controller, which is an external entity to SimMobility (presented in detail in section 3.3).

\section{**Figure 1: SimMobility MidTerm (MT) structure**}

\subsection{Flexible Mobility On-Demand Framework}

In order to facilitate the study of complex interactions between independent drivers, the central controller and travelers' decisions, we propose an event-based modelling framework. Here, drivers, controllers, and travelers are represented as separate decision agents with decisionmaking triggered by specific events. In the next sections we will describe each of these three agents in our proposed framework, their decision-making dimensions and their trigger events. It is worth noting that for the service drivers, the richest set of decision dimensions is that of the traditional MoD driver, i.e. the (almost independent) taxi driver. Therefore, we will first describe this set of decisions which will later be modified and extended to accommodate other MoD driver behavior, such as ride-hailing and shared services.

\subsection{Traveler Agent}

As part of the Day Activity Schedule (DAS) generated by Pre-day, an agent has an MoD mode assigned to her, as well as start and end time of activity and exact location. When the time of simulation reached its $\mathrm{MoD}$ journey starting time, she will either (1) start searching for a MoD driver on the street either by hailing or by walking to the closest MoD stand or (2) request an $\mathrm{MoD}$ and wait for pick-up. Her request is added to a first in, first out array of potential clients for a MoD at that link and waits for the MoD acceptance. As for the Uber, and Lyft-like traveler, its meeting point with the driver will be at her home or activity location. The Uber, and Lyft-like traveler cannot be picked up at the MoD stand.

\subsection{Mobility On-Demand Driver Agent}

The traditional MoD-drivers are modelled as agents with their own preferences. They can choose their next move and their next client. Their interactions with the travelers is a result of driver's 
choice. For the traditional MoD driver, we embed in the drivers the knowledge about the historical space and time distribution of the clients, based on historical demand data. The information is then used by MoD drivers to choose the most adequate MoD stands to stop at different hours of the day and choose the most attractive routes for finding clients in the street.

\subsubsection{State Vector of the MoD Driver}

An MoD driver is at any moment in one state of the state vector. To allow for different MoD services, the proposed state vector is composed of the following Boolean variables:

Booked (B) A MoD driver is on its way to pick up a customer. The MoD vehicle is booked after it interacts with the controller

Occupied (O) A client is in the MoD vehicle, and the MoD driver is heading towards the client's destination.

Queueing $(\boldsymbol{Q})$ The MoD driver is in a queue at a MoD stand to pick up a client.

Cruising $(\boldsymbol{C}) \quad$ A MoD driver is searching the streets to find the next client. More generally, it represents that the vehicle is ready to take a passenger.

Direct-to- The variable describes a state where the MoD driver is direct to a specific destination (D) destination. She will not make intermediate stops or pickups until she reaches the destination.

Break (K) The MoD driver is on a break; the driver temporarily will not be available to accept any kind of requests.

\subsubsection{Events influencing the MoD driver agent}

The designed framework is event driven and there are seven major event types that influence the MoD driver agent as follows:

Pick-up event: This event occurs when the MoD driver picks up a traveler. The pick-up can happen on-road or at any stopping bay; we treat different bays as a MoD stand.

Drop-off event: This event occurs when the MoD reaches the destination of the traveler. After this event, the MoD-driver re-evaluates her strategy.

Join queue event: This event occurs when the MoD driver reaches the MoD-stand. The MoD driver can join the queue at the stand only if the number of drivers already queueing at the stand is below capacity.

Controller request event: This event describes the messages the controller sends to the $\mathrm{MoD}$ driver regarding the pick-up request. This event can occur at any time.

Cruising for too long event: This event is triggered when the MoD driver has been cruising unsuccessfully for too long. Afterward, the MoD driver will choose "do not cruise."

Queuing for too long event: This event is triggered when the MoD driver has been queueing unsuccessfully for too long at a MoD-stand. After this event, the MoD driver re-evaluates her strategy.

Figure 2 describes the traditional MoD driver behavior framework, showing the decision models and choices. The simulation starts by loading the agents at their home location as defined in the synthetic population. The MoD driver's initial decision will be to work or to take a break; if the driver decides to take a break, her state will change to break activity whose duration is predetermined. Location and time information will be kept in the simulation. 


\section{**Figure 2: Traditional MoD driver behavior framework**}

If the MoD driver chooses to cruise, she will search the streets to find a client. She can do so by moving towards a predetermined zone, then the zone-based cruising model will be activated first and the route choice model will be activated second. After reaching the desired zone, the MoD driver will cruise randomly from link to link with no specific target. The MoD will choose the desirable zone according to the historical demand. If a client is found, the MoD driver will pick her up; the route choice model will be activated, and the MoD will move towards the client destination. If the MoD driver is cruising for too long, she will change her state to "do not cruise," else, she will go to her initial decision of whether to take a break.

In the case of a coordinated ride-hailing driver behavior framework, majority of the decisions are taken out so that the driver cannot: (1) choose whether to cruise or not to cruise (2) choose to go to a MoD stand, queue, and pick up a passenger there (3) choose to pick up passengers on the street, or (4) choose her next zone to in which to cruise. On the interaction between the driver and the MoD controller see Section 3.5. Note that traveler's choices are not modeled in detail and will be incorporated in future research.

\subsection{MoD Controller: the case of Automated MoD}

The model of Automated Mobility on Demand (AMoD) builds upon our previous work (5), extended to handle new capabilities, for instance parking, handling single or shareable ride requests, etc. An AMoD service consists of a fleet of vehicles and a controller. The interactions between the user, the controller and the vehicles are depicted in Fig. 3. Users send trip requests to the controller, which assigns them to vehicles in the form of schedules. The controller computes and continuously updates a schedule for each vehicle, which dictates the sequence of operations, i.e., pick-up, drop-off, etc., that the vehicle will perform. It is worth emphasizing the main difference between MoD and AMoD services: the former are driven by the choices and the experience of the drivers, while the latter are fully determined by a centralized controller and vehicles follow the instructions given in the schedule.

\section{User requirements}

In the trip request, the user specifies pick-up and drop-off locations, shareability, indicating whether the user is willing to share her ride with other users or not, maximum waiting time she is willing to accept and the tolerated delay at arrival, i.e., the amount of additional delay she can accept with respect to the minimum travel time possible. With the tolerated delay, the user declares an upper bound of the delay she is willing to accept. In brief, we call time constraints of a trip request its maximum waiting time and tolerated extra delay.

\section{**Figure 3: Interactions between agents in the model of AMoD service**}

\section{Controller-Vehicle interaction}

As explained before, the activities of the vehicles are completely determined by the centralized controller, by means of schedules computed by the controller and sent to vehicles. At any time, each vehicle has a schedule, which is a sequence of commands that can be of the following types: 
- Pick-up a user; it includes the user-ID and the related trip-request containing all the user requirements.

- Drop-off a user: similar to the pick-up command.

- Cruise to a certain zone.

- Go to park at a certain node.

The commands in a schedule can be arranged in any plausible order ensuring, for example, that a drop-off for any user comes after the respective pick-up and that the number of passengers never exceeds the number of seats available in the vehicle

\section{Schedule Computation}

The controller continuously collects request from users and periodically, i.e., every 10 seconds, computes or updates vehicle schedules in order to match them. The controller computes feasible schedules. A schedule is feasible if (i) all its pick-ups can be performed respecting the maximum waiting time specified in the respective trip request and (ii) all its drop-offs can be performed respecting the tolerated extra-time specified in the respective trip request. Note that if a feasible schedule is updated inserting the pick-up and the drop-off of a new user, in order to ensure that the new schedule is still feasible, not only we have to check that the time constraints of new user are met, but we have also to consider that the insertion of the new pick-ups and drop-offs may imply a detour for the vehicle that can delay the pick-up of the drop-offs of the passengers previously inserted in the schedule, possibly violating their time constraints. If a modified schedule is unfeasible, the modification is not accepted, i.e., the new passenger cannot be served by that vehicle and the controller will attempt to match her to another vehicle. The controller is able to handle both shareable and non-shareable requests. To do so, first the shareable requests are matched to the available vehicles with some available seats, using the insertion heuristic detailed in (5). Then, the controller matches the non-shareable requests with the closest empty vehicles. Finally, updated schedules are sent to the vehicles.

\section{CASE STUDY: FROM TRADITIONAL TO AUTOMATED MOBILITY ON DEMAND IN SINGAPORE}

Researchers who have focused on the algorithms to optimize fleet operation, have claimed the superiority of $\mathrm{AMoD}$ over $\mathrm{MoD}$ and supported the assumption that $\mathrm{AMoD}$ will improve urban mobility. While research has produced its claims overlooking the effect of congestion and an accurate model of drivers, the second side has been represented, with few exceptions, by conceptual or economic reasoning, with lack of quantifiable results. To fully understand the impact of shifting from current human-driven MoD to future Automated MoD, we claim it is necessary to accurately model driver behavior, which has been overlooked in current studies on AMoD. We demonstrate the validity of this claim by comparing accurately-modeled traditional $\mathrm{MoD}$ service and future AMoD in Singapore.

\subsection{Model Estimation}

For the estimation of each of the driver decision dimensions, a GPS dataset collected from a major MoD fleet operator in Singapore in 2013 was used. This data set contains more 25 million records each day for a period of 30 days, and containing vehicle number, time, position and service status data. MoD fleet size was fixed according to the information provided by the Land 
Transport Authority in Singapore (19). The simulation is focused in reproducing MoD driver's behaviors in a typical working day in a city. MoD driver agents are identified by synthetic population generated for Singapore for 2012. Their start time of work and shift duration was modeled by fitting a distribution based on MoD GPS data, which was then used to determine each driver's starting time and shift duration. In Figure 4 (a) and (b) the distribution of shift duration and shift starting time, as obtained using the GPS traces, is presented (in blue).

The specific behaviors of the traditional MoD drivers are modelled using a discrete-choice framework. Five are estimated, namely: (i) Break Model; (ii) Cruising Model; (iii) Stand Choice Model; (iv) Zone Based Cruising Model; and (v) Route Choice Model.

**Figure 4: The distribution of (a) shift duration and (b) shift starting time**

\section{Break Model}

The break model was estimated as a binary logit model estimating the probability of a MoD driver to take a break. A subsample of 39,831 observations taken from the GPS dataset were used for the model estimation. Table 1 shows the estimation results of the break model. The directions of the effects of all variables are theoretically consistent. $86 \%$ of breaks take place outside the CBD and 50\% of the drivers take a break within 5 hours from their previous one.

\section{Spatial Choice Model: Stand Choice}

The second model is for simulating the choice of a stand. It is estimated as a location choice model using the multinomial logit formulation for the probability of a MoD driver to choose a specific stand from 214 alternative stations scattered around in the network. We used a subsample of 2,324 observations taken from the GPS dataset. Table 1 presents the parameter estimates for the stand choice model. The directions of the effects of all variables are theoretically reasonable, with an estimated marginal effect of travel cost per kilometer of 0.24.

\section{Integrated Spatial Choice: Zone Based Cruising and Route Choice Model}

We estimated a multi-level decision model for the cruising zone selection and the route selection to reach the zone, using the nested logit formulation. The model was estimated by the sequential estimation procedure. At the upper level of this model, the probability to choose a specific zone to cruise in among all 1169 traffic zones, is estimated while at the lower level, a specific route to reach this zone is chosen. The data used were a subsample of 4000 observations taken from the GPS dataset. As the number of alternatives is very large, the $\rho^{2}$ is small as expected.

Surprisingly, many highly visited cruise zones are not in the CBD. In fact, the most visited zone is outside the CBD with more than 1,580 pickups.

\section{Cruise Choice Model}

The cruise/no cruise choice model was estimated as a binary logit model, which estimates the probability of a MoD driver to cruise. The data used were a subsample of 13,742 observations taken from the GPS dataset. Table 1 presents the parameter estimates for this model. The directions of the effects of all variables are theoretically reasonable. Interestingly, it was found that as the employment density increases, the driver is less likely to cruise. In Singapore, the high employment areas are also characterized by a large number of stands which makes the search for customers easy. Overall $30 \%$ of the drivers choose to go to a stand while the rest choose to cruise. 


\section{**Table 1: The MoD driver behavior model estimates**}

Cruising for too long, and the queueing for too long are handled by drawing a unique value for each driver from the distribution obtained using the GPS data. In Figure 5, the distribution of cruising durations, and queuing durations as obtained using the GPS traces, is presented (in blue) as well as a log-normal curve that was fitted (in red).

**Figure 5: The distribution of (a) cruising duration, and (b) queuing duration**

\subsection{Study Area}

Our study was conducted using a representative synthetic population and network of Singapore. The total area of Singapore is $721.5 \mathrm{~km}^{2}$ with a population of 5.3 million individuals in 2012 (20). In Singapore, passengers make over 8 million trips on a daily basis with an average stop rate of 1.5 per individual. Singapore has a developed transportation system covering $3,356 \mathrm{~km}$ of roads which includes 10 expressways. The public transportation system consists of $15 \mathrm{MRT}$ and LRT lines with a total of 124 subway stations (92 MRT stops and 32 LRT stops) and 728 bus lines spanning the island with a total of 4607 bus stops. The road network consists of 6220 nodes (intersections), 30585 segments (road sections with homogeneous geometry) and 14799 links (groups of one or more segments with similar properties). Singapore Island is divided into 1169 Traffic Analysis Zones (TAZs).

\subsection{Experimental Design}

Two different scenarios are designed and simulated. We consider a "traditional MoD only" scenario where traditional MoD is operated for on-demand service delivery. The available modes are single occupancy car (Car), sharing with one extra passenger (Car Sharing 2), sharing with two extra passengers (Car Sharing 3), Private Bus, public Bus, Mass Rapid Transit (MRT), Motorcycle, Walk and traditional MoD. The modal availabilities are in accordance with our study area, which we describe in the following section. In the second scenario we introduce automated MoD, replacing traditional MoD service with two new modes: AMoD as a nonshared, driverless ride (AMoD), and $\mathrm{AMoD}$ as a shared ride (AMoD Pool), while the availability of all other modes from the base case scenario stays the same. We refer to this scenario as "AMoD only" scenario. In order to generate the demand for AMoD modes, given the absence of appropriate data, we assumed that individual preference towards AMoD is similar to that of MoD with some modifications. The first set of assumptions is that a single AMoD ride will be $50 \%$ cheaper compared to $\mathrm{MoD}$, and that a shared ride will be $30 \%$ cheaper than a single ride (21). We also implemented a distance-based additional in-vehicle travel time for the passengers who share the vehicle with other passengers (based on current Uber data). Furthermore, we have added the expected additional waiting time for the AMoD Pool rider. We conduct morning peak (6-10 am) simulations for each scenario in SimMobility using the Singapore network. The results are summarized in the following section. We also compare the impacts of fleet size and parking strategies for AMoD on network performance. 
1

\section{RESULTS AND DISCUSSION}

The mode share distribution for each scenario, and the demand locations for MoD and $\mathrm{AMoD}$ are shown in Figure 6. In the "traditional MoD only" case, MRT and Public Bus (PT) account for $48.6 \%$ of the share, while Walk is about $20 \%$ of the share, and Private Bus $10 \%$. Private car accounts for $12 \%$ of the share, and an additional $4.3 \%$ will share the Car with other passengers. The traditional MoD service consists of $3 \%$ of the share. In the "AMoD only" scenario, where $\mathrm{AMoD}$ services are offered as a substitute for traditional MoD services, we see significant reduction in PT mode share of more than $6 \%$, with a shift towards $\mathrm{AMoD}$, which consists of 13.2\%. We also observe a small reduction in Walk and Private Bus, as well as in Car Sharing modes, while there is no change in Car mode share.

**Figure 6: Mode share and demand profile during morning peak for (a) Traditional MOD and (b) Automated Mobility on Demand services**

Fig. 6 shows the activity of the traditional MoD drivers under the models specified in section 4 . As expected, the number of drivers queueing at the MoD stands and cruising reduces during peak hours. On average, drivers spend 8 minutes cruising and 2 minutes queueing during the morning peak.

\section{**Figure 7: MoD driver behavior during simulation**}

In Fig. 8, we observe the performance of the controller with regard to trip request satisfaction under various fleet loadings. Based on these results and on those in Figure 10, the 45,000-fleet case appears as the user-optimal case. In order to compare to the alternate strategy wherein vehicles are cruising, instead of parked, we compare the 36,000-fleet case under both conditions. Comparing Figures $7 \mathrm{c}$ and $7 \mathrm{~d}$, we see that waiting time is slightly lower under the parking strategy (as indicated by the area between the blue and orange curves). Overall, parking ensures that demand is ultimately met, as can be seen toward the end of the curves in both Fig $7 \mathrm{c}$ and $7 \mathrm{~d}$. Note that Fig. 7 counts the requests, pick-ups and drop-offs observed in each 5-minute interval. Obviously, not all the requests sent in a certain 5-minute interval are picked up and dropped off in the same interval, as they will be served later. The request-to-dropoff ratio is highest at about 8:00am and narrows down toward the end of the observed scenario. When the service is not well configured, e.g. with 36,000 vehicles and no parking, the number of requests served do not recover the number of requests sent, while using parking solves this problem.

\footnotetext{
**Figure 8: Controller performance for AMOD service under various fleet size scenarios, all implemented with parking except $(\mathbf{d}) * *$
} 
In Figure 8 we compare driver behavior and fleet management between the "Traditional MoD only" and the "AMoD only" scenarios. Note that MoD vehicles are not parked or driven to a prespecified location to pick up. Instead, passengers are picked up while hailing or waiting at the taxi stand. In the AMoD case, the fleet is more efficiently managed, as they are introduced incrementally, while MoD drivers are introduced according to their shift starting time. As the MoD shifts are set inefficiently, they spend a lot of time cruising. However, this inefficiency is greatly reduced towards the end of peak period.

\title{
**Figure 9: Vehicle utilization profiles for "Traditional MoD only" (20,000 vehicles) and best-case "AMoD only" (45,000 vehicles) scenarios**
}

\begin{abstract}
**Figure 10: Waiting time (W) across automated on-demand modes for the different scenarios (in minutes). Avg Network-Wide $W$ is the average $W$ over buses, trains and MoD. Journey Time captures average travel time across all modes**
\end{abstract}

In Fig. 9 we report the impact on user-metrics of the different services, under different settings. Journey times (JT) do not include waiting times (W), which are represented separately. As expected, the shared requests experience higher waiting times. The increase in fleet size is clearly beneficial for non-shared requests, while its impact is less pronounced for the shared. This can be explained by the interest of the operator in decreasing the miles traveled: even if a large fleet is available, it will try to serve the shared requests with as few active vehicles as possible, as long as the user requirements are satisfied. This is reflected in the matching algorithm we have used (5). It is clear from Fig. 9 that the 45,000 AMoD fleet case is useroptimal. A limitation of the simulation framework at the time this experiment was carried out is that taxi waiting times are not given explicitly. However, as can be seen from the average journey times, similar levels of service are provided by both the AMoD and the traditional MoD cases. In Table 2, we compare average travel times and distances for passenger-vehicle trips for the two scenarios. These indicate that the overall patterns for single-passenger trips are largely unchanged. As expected, travel times for AMoD Pool are about 50\% greater on average.

**Table 2: Average travel times and distances for passenger-vehicle trips in both scenarios**

\section{CONCLUSIONS}

We have demonstrated an agent-based simulation of daily activity patterns and movements in a dense urban network, using Singapore as a case study. Importantly, we have modeled in adequate fashion the behavior and movement of traditional mobility on demand services and compared comprehensively how this performs relative to the near-futuristic automated mobility on demand service. Our simulator - SimMobility - has enabled us to test the impacts on network performance of the user optimal automated case to the traditional case. 
A key finding of this research is that a significant reduction in PT modes (12\%) and a dramatic increase in mobility on-demand share (four times greater) are observed when AMoD services are offered as a substitute for traditional MoD services. Additionally, while demand for AMoD is over four times greater than that of traditional MoD, only about twice as many fleet are required to satisfy the increased demand levels. In the "traditional MoD only" case, there are 20,000 taxis available during the morning peak. For the "AMoD only" case, we employed simulation tests to obtain the user optimal fleet size of 45,000 for the same period, servicing 0.2 million trips (over 5 times those of traditional MoD). This demonstrates that AMoD fleet are more efficiently managed compared to MoD fleet, and have the potential to improve urban mobility outcomes with the same level of service, at likely lower costs. We also show that when ridesharing is predominant, then fewer fleet can serve the demand just as efficiently.

Our further avenues of research include quantifying the cost implications of AMoD implementation strategies and comparing their respective benefits. Given our capabilities to sufficiently model current Uber-like mobility-on-demand systems, we would like to further investigate driver behavior under today's MoD frameworks.

The differences in urban form and rates of technological and infrastructural development indicate that the introduction of AMoD services will have widely varying impacts. There are critical questions to be answered regarding its effects on congestion, parking and public transportation ridership. In order to better understand these future patterns, we are currently developing prototype cities representing distinct urban typologies. By simulating relevant scenarios in these prototype cities, we can further obtain insights into policy intersections for the best outcomes for AMoD service implementation.

\section{AUTHOR CONTRIBUTION STATEMENT}

The authors confirm contribution to the paper as follows: study conception and design: B. Nahmias-Biran, J. B. Oke, A. Araldo, K. Basak, R. Seshadri, A. Akkinepally, C. L. Azevedo, M. Ben-Akiva; analysis and interpretation of results: B. Nahmias-Biran, J. B. Oke, N. Kumar, A. Araldo, K. Basak, C. L. Azevedo; draft manuscript preparation: B. Nahmias-Biran, J. B. Oke, K. Basak, C. L. Azevedo. All authors reviewed the results and approved the final version of the manuscript.

\section{ACKNOWLEDGMENTS}

This research was supported by the National Research Foundation Singapore through the Singapore MIT Alliance for Research and Technology's Future Urban Mobility Interdisciplinary Research Group. The authors would like to thank their colleagues and collaborators in the Intelligent Transportation Systems Lab both at the Singapore-MIT Alliance for Research and Technology (SMART) and at the Massachusetts Institute of Technology (MIT) for both technical support and theoretical insights over the course of this effort. The authors also thank the anonymous reviewers for their time and attention in helping us to improve the quality of this manuscript.

\section{DISCLAIMER}

45 The contents of this report reflect the views of the authors who are responsible for the facts and

46 accuracy of the data presented herein. The contents do not necessarily reflect the official views 
or policies of Ministry of Transport (MoT), Land Transport Authority (LTA), Urban

Redevelopment Authority (URA) and Housing Development Board (HDB) in Singapore.

\section{REFERENCES}

1. Salanova, J. M., Estrada, M., Aifadopoulou, G., Mitsakis, E. (2011). A review of the modeling of taxi services. Procedia-Social and Behavioral Sciences, 20, 150-161.

2. Martinez, L. M., Correia, G. H., Viegas, J. M. (2015). An agent-based simulation model to assess the impacts of introducing a shared-taxi system: an application to Lisbon (Portugal). Journal of Advanced Transportation, 49(3), 475-495.

3. Cheng, S. F., Nguyen, T. D. (2011, August). Taxisim: A multiagent simulation platform for evaluating taxi fleet operations. In Proceedings of the 2011 IEEE/WIC/ACM International Conferences on Web Intelligence and Intelligent Agent TechnologyVolume 02 (pp. 14-21). IEEE Computer Society.

4. Adnan, M., F. C. Pereira, C. M. L. Azevedo, K. Basak, M. Lovric, S. Raveau, Y. Zhu, J. Ferreira, Z. Christopher, and M. E. Ben-Akiva (2016). SimMobility: A multi-scale integrated agent-based simulation platform. In Transportation Research Board 95th Annual Meeting, 2016, 16-2691.

5. Basu, R., Araldo, A., Akkinepally, A. P., Nahmias Biran, B. H., Basak, K., Seshadri, R., Deshmukh, N., Kumar, N., Azevedo, C. L., Ben-Akiva, M. (2018). Automated Mobilityon-Demand vs. Mass Transit: A Multi-Modal Activity-Driven Agent-Based Simulation Approach. Transportation Research Record.

6. Maciejewski, M., Salanova, J. M., Bischoff, J., Estrada, M. (2016). Large-scale microscopic simulation of taxi services. Berlin and Barcelona case studies. Journal of Ambient Intelligence and Humanized Computing, 7(3), 385-393.

7. Hörl, S. (2016). Implementation of an autonomous taxi service in a multi-modal traffic simulation using MATSim (Master's thesis, Chalmers University of Technology and University of Gothenburg).

8. Lee, D. H., Wu, X. (2013). Dispatching strategies for the taxi-customer searching problem in the booking taxi service. In Proceedings of the Transportation Research Board 92nd Annual Meeting.

9. Alonso-Mora, J., Samaranayake, S., Wallar, A., Frazzoli, E., Rus, D. (2017). On-demand high-capacity ride-sharing via dynamic trip-vehicle assignment. Proceedings of the National Academy of Sciences, 114(3), 462-467

10. Lee, D. H., Wang, H., Cheu, R., Teo, S. (2004). Taxi dispatch system based on current demands and real-time traffic conditions. Transportation Research Record: Journal of the Transportation Research Board, (1882), 193-200.

11. He, F., Shen, Z. J. M. (2015). Modeling taxi services with smartphone-based e-hailing applications. Transportation Research Part C: Emerging Technologies, 58, 93-106.

12. Al-Ayyash, Z., Abou-Zeid, M., Kaysi, I. (2016). Modeling the demand for a shared-ride taxi service: An application to an organization-based context. Transport Policy, 48, 169182.

13. Wong, R. C. P., Szeto, W. Y., Wong, S. C., Yang, H. (2014). Modelling multi-period customer-searching behaviour of taxi drivers. Transportmetrica B: Transport Dynamics, 2(1), 40-59. 
14. Yang, C., Gonzales, E. J. (2016). Modeling vacant yellow taxi customer search behavior in a holiday week in New York City (No. 16-6850).

15. Atasoy, B., Ikeda, T., Ben-Akiva, M. E. (2015). Optimizing a flexible mobility on demand system. Transportation Research Record, (2536), 76-85. large-scale taxi traces. PLoS ONE, 11(11), e0165597.

17. Zhang, Y., Li, B., \& Ramayya, K. (2016). Learning individual behavior using sensor data: The case of GPS traces and taxi drivers.

18. Ji, Y., Du, Y., Liu, Y., Zhang, H. M. (2016). Empirical Behavioral Study of AirportServing Taxi Drivers Using Automatic Vehicle Location Data. Journal of Urban Planning and Development, 143(1), 04016026.

19. Land Transport Authority, (2018). Annual Vehicle Statistics 2018. Government of Singapore. Retrieved April 2019. https://www.lta.gov.sg/content/dam/ltaweb/corp/PublicationsResearch/files/FactsandFigu res/MVP01-1_MVP_by type.pdf

20. Singapore Department of Statistics, (2018). Yearbook of Statistics Singapore. Retrieved September 2019. https://www.singstat.gov.sg//media/files/publications/reference/yearbook_2018/yos2018.pdf

21. Chia, J. (2017). Revisiting the Sharing Economy in Singapore. Working document. Lee Kuan Yew School of Public Policy at the National University of Singapore. https://lkyspp.nus.edu.sg/docs/default-source/case-studies/revisiting-the-sharingeconomy-(updated-092017).pdf?sfvrsn=eaa8950b_0 


\section{List of figure captions:}

Figure 1: SimMobility MidTerm (MT) structure

Figure 2: Traditional MoD driver behavior framework

Figure 3: Interactions between agents in the model of AMoD service

Figure 4: The distribution of (a) shift duration and (b) shift starting time

Figure 5: The distribution of (a) cruising duration, and (b) queuing duration

Figure 6: Mode share and demand profile during morning peak for (a) Traditional MOD and (b) Automated Mobility on Demand services

Figure 7: MoD driver behavior during simulation

Figure 8: Controller performance for AMOD service under various fleet size scenarios, all implemented with parking except (d).

Figure 9: Vehicle utilization profiles for "Traditional MoD only" (20,000 vehicles) and best-case "AMoD only" (45,000 vehicles) scenarios

Figure 10: Waiting time (W) across automated on-demand modes for the different scenarios (in minutes). Avg Network-Wide $W$ is the average $W$ over buses, trains and MoD. Journey Time captures average travel time across all modes.

\section{List of table titles:}

Table 1: The MoD driver behavior model estimates

Table 2: Average travel times and distances for passenger-vehicle trips in both scenarios 


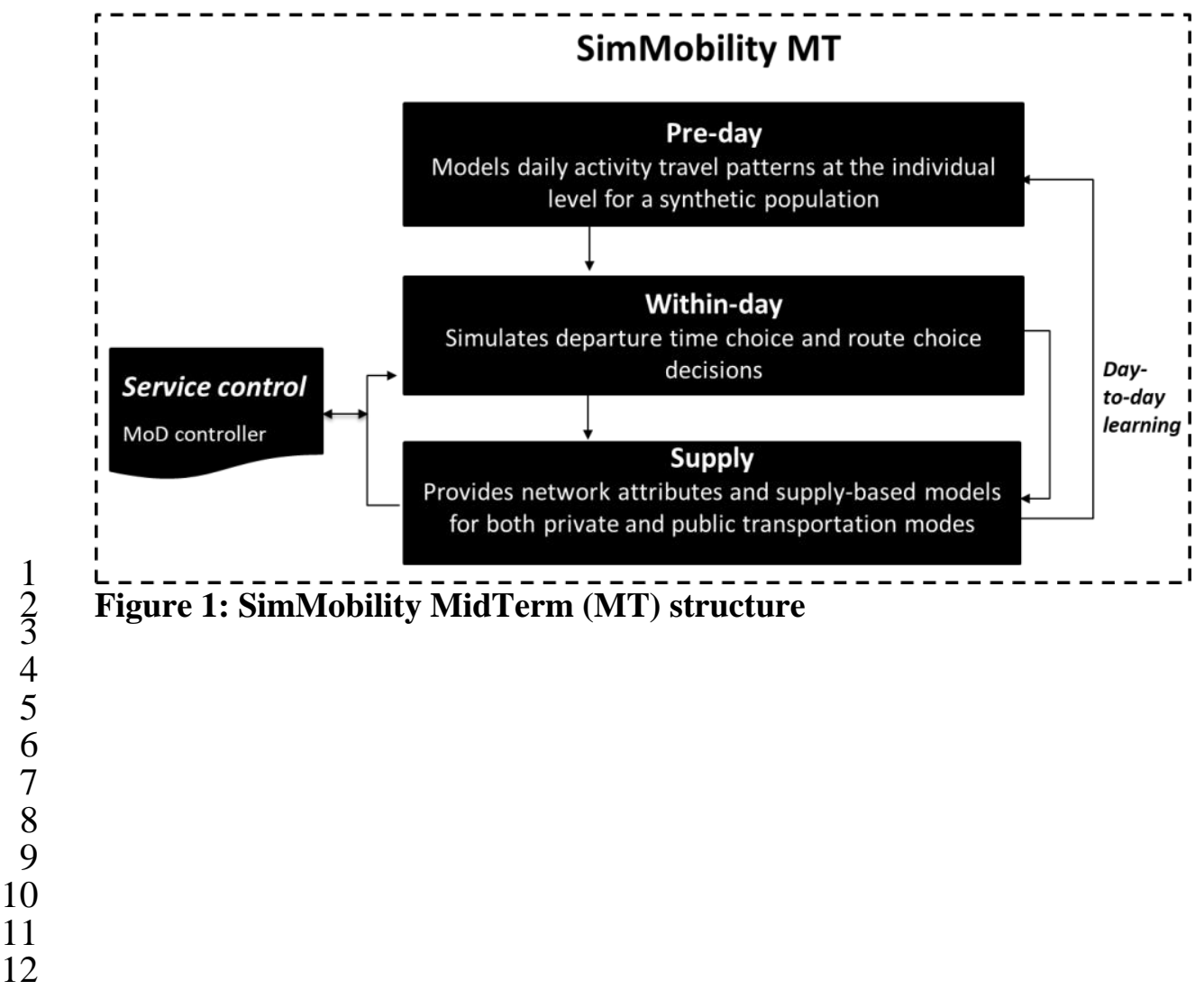




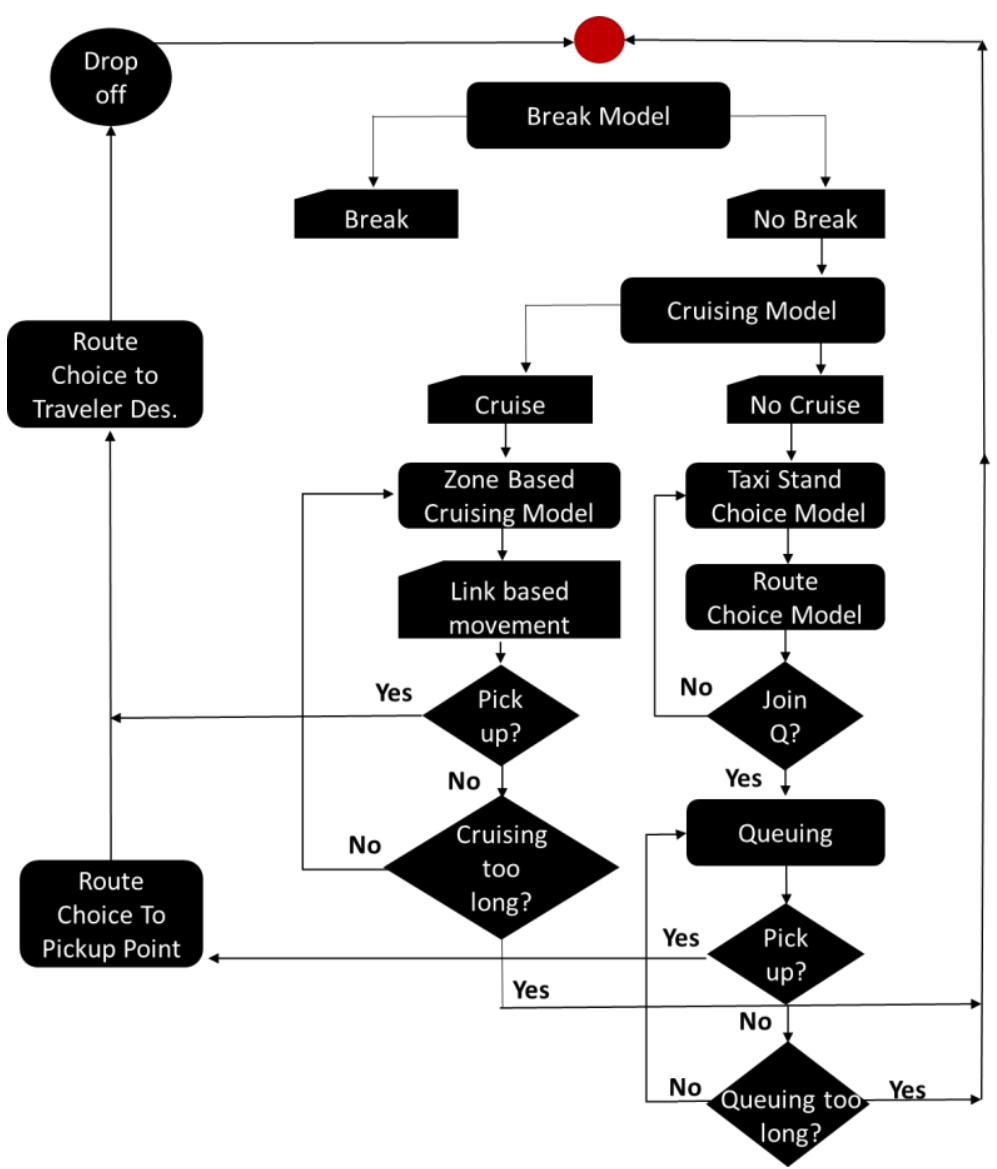

Figure 2: Traditional MoD driver behavior framework 


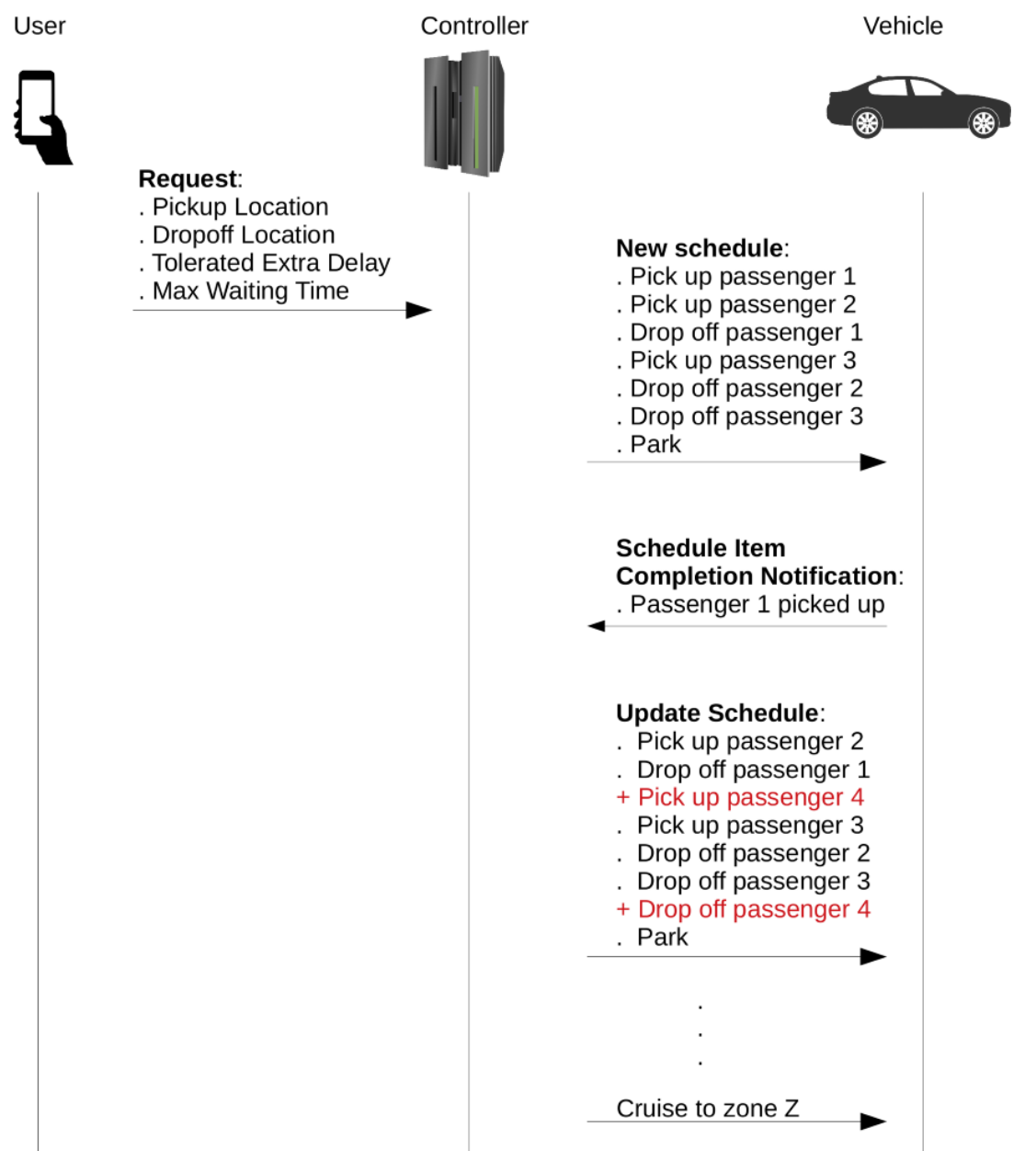



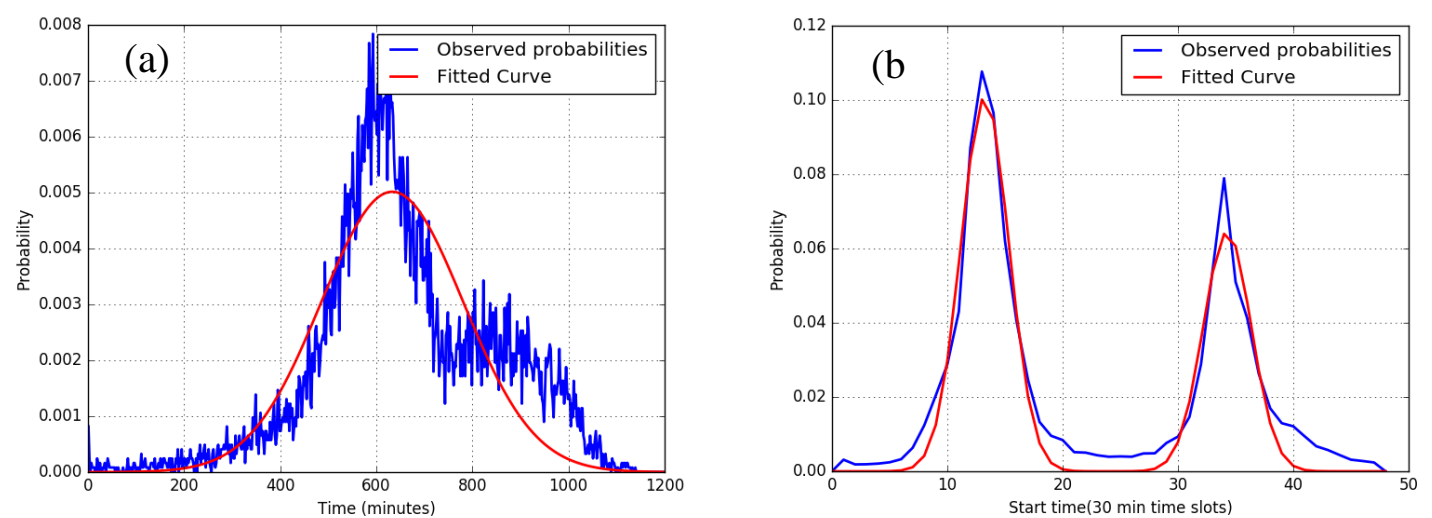

Figure 4: The distribution of (a) shift duration and (b) shift starting time 
Table 1: The MoD driver behavior model estimates

\begin{tabular}{|c|c|c|c|c|}
\hline \multicolumn{5}{|l|}{ Break model } \\
\hline Variable & Coefficient & $\begin{array}{l}\text { Asymptotic } \\
\text { st. error }\end{array}$ & $t$ statistic & Summary statistics \\
\hline Break constant & -3.03 & 0.0457 & -66.28 & \multirow{2}{*}{$\begin{array}{l}\text { Num. of observations } \\
=39,831\end{array}$} \\
\hline In CBD dummy & -0.47 & 0.0451 & 10.43 & \\
\hline Log employment & 0.19 & 0.0165 & 11.5 & $\mathscr{L}(0)=-27608.74$ \\
\hline $\begin{array}{l}\text { Travel cost to the nearest stand } \\
\text { (SGD) }\end{array}$ & -0.473 & 0.0744 & -6.36 & $\mathscr{L}(\beta)=-14377.43$ \\
\hline $\begin{array}{l}\text { Time passed from the last break } \\
\text { (hr) }\end{array}$ & 0.0633 & 0.00343 & 18.44 & $\rho^{2}=0.479$ \\
\hline $\begin{array}{l}\text { Time left to the end of the shift } \\
\text { (hr) }\end{array}$ & 0.0557 & 0.00402 & 13.84 & \\
\hline Number of previous breaks & -0.365 & 0.00787 & 46.32 & \\
\hline \multicolumn{5}{|l|}{ Stand choice model } \\
\hline Variable & Coefficient & $\begin{array}{l}\text { Asymptotic } \\
\text { st. error }\end{array}$ & $t$ statistic & Summary statistics \\
\hline Break constant & 2 & 0.0896 & 22.37 & \multirow{2}{*}{$\begin{array}{l}\text { Num. observations }= \\
2324\end{array}$} \\
\hline In CBD dummy & -1.24 & 0.0944 & -13.19 & \\
\hline Log employment & -0.299 & 0.0212 & -1.41 & $\mathscr{L}(0)=-12254.165$ \\
\hline $\begin{array}{l}\text { Travel cost to the nearest stand } \\
\text { (SGD) }\end{array}$ & -5.02 & 0.432 & -11.88 & $\mathscr{L}(\beta)=-5720.485$ \\
\hline $\begin{array}{l}\text { Time passed from the last break } \\
\text { (hr) }\end{array}$ & 1.08 & 0.188 & -5.72 & \multirow[t]{3}{*}{$\rho^{2}=0.533$} \\
\hline $\begin{array}{l}\text { Time left to the end of the shift } \\
\text { (hr) }\end{array}$ & 1.75 & 0.0674 & 25.94 & \\
\hline Number of previous breaks & 2.31 & 0.0792 & 29.16 & \\
\hline \multicolumn{5}{|c|}{ Integrated spatial choice and route choice model } \\
\hline Variable & Coefficient & $\begin{array}{l}\text { Asymptotic } \\
\text { st. error }\end{array}$ & $t$ statistic & Summary statistics \\
\hline In CBD dummy & 1.38 & 0.0748 & 18.41 & \multirow{2}{*}{$\begin{array}{l}\text { Num. of observations } \\
=4999\end{array}$} \\
\hline Stand dummy & 0.633 & 0.0427 & 14.81 & \\
\hline Route choice logsum & 0.00313 & 0.000452 & 6.93 & $\mathscr{L}(0)=-34924.773$ \\
\hline Log scale parameter & 0.799 & 0.0102 & 78.6 & $\mathscr{L}(\beta)=-31192.34$ \\
\hline Zone's area $\left(\mathrm{km}^{\wedge} 2\right)$ & 2.38 & 0.175 & 13.59 & $\rho^{2}=0.107$ \\
\hline \multicolumn{5}{|l|}{ Cruise choice model } \\
\hline Variable & Coefficient & $\begin{array}{l}\text { Asymptotic } \\
\text { st. error }\end{array}$ & $t$ statistic & Summary statistics \\
\hline Cruise constant & 0.639 & 0.0468 & 13.65 & \multirow{2}{*}{$\begin{array}{l}\text { Num. of observations } \\
=13742\end{array}$} \\
\hline $\begin{array}{l}\text { Time left to the end of the shift } \\
\text { (hr) }\end{array}$ & 0.0144 & 0.00436 & 3.31 & \\
\hline In CBD dummy & -0.354 & 0.0541 & -6.53 & $\mathscr{L}(0)=-9525.229$ \\
\hline Total pick up at the stand & -0.147 & 0.0403 & -3.64 & $\mathscr{L}(\beta)=-7624.537$ \\
\hline Employment density $\left(1 / \mathrm{km}^{\wedge} 2\right)$ & -0.0314 & 0.00329 & -9.52 & $\rho^{2}=0.200$ \\
\hline Travel distance to stand (km) & 0.595 & 0.0373 & 15.95 & \\
\hline
\end{tabular}



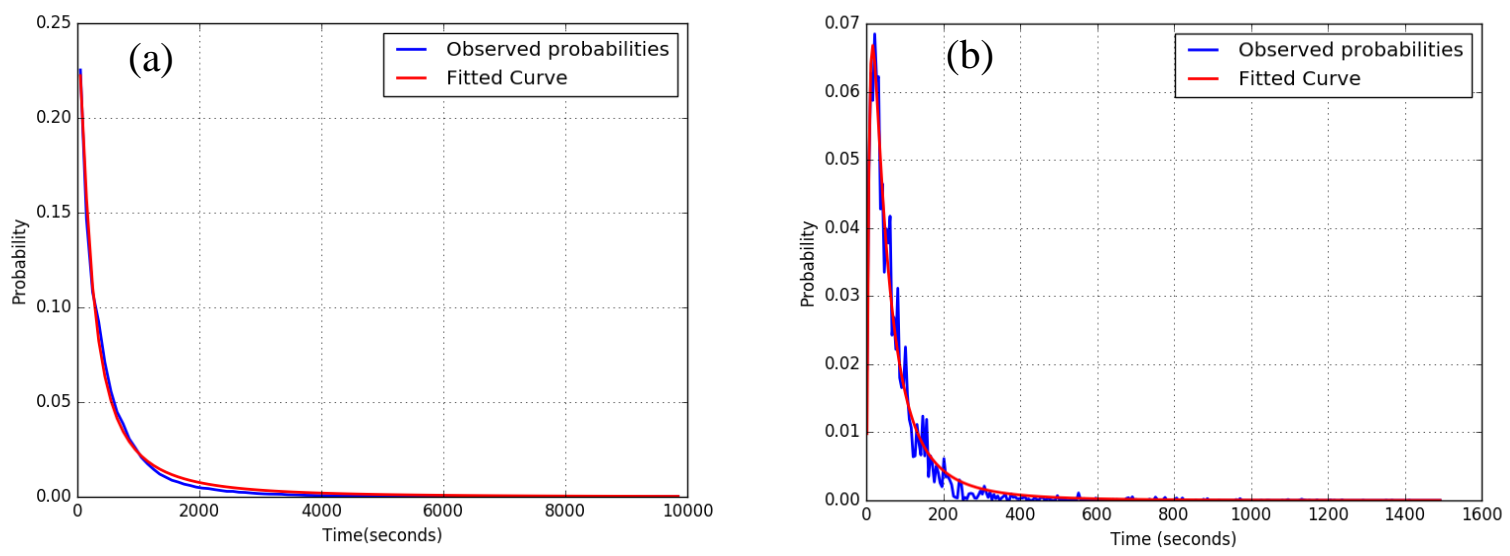

Figure 5: The distribution of (a) cruising duration, and (b) queuing duration 

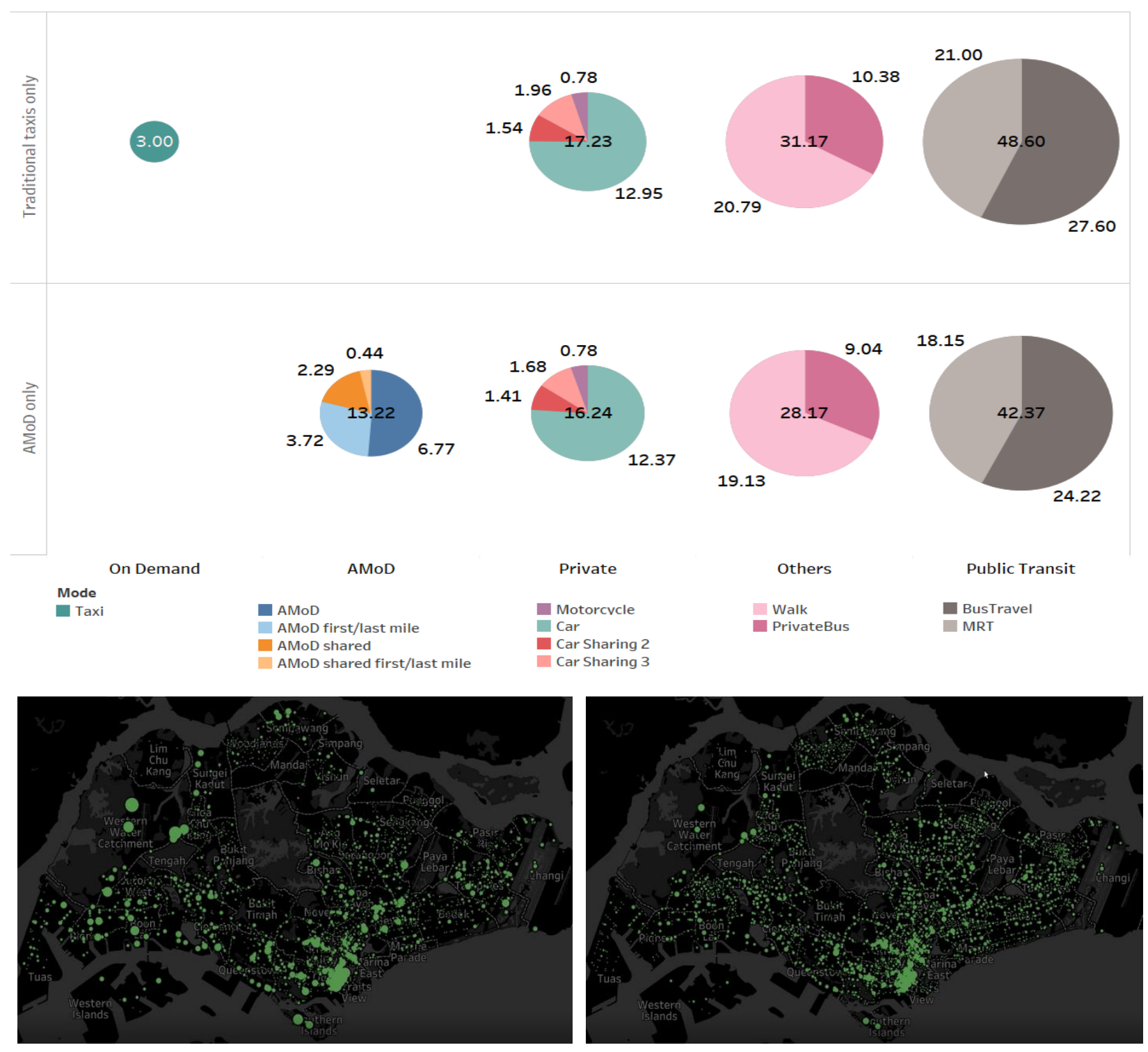

Figure 6: Mode share and demand profile during morning peak for (a) Traditional MOD and (b) Automated Mobility on Demand services 

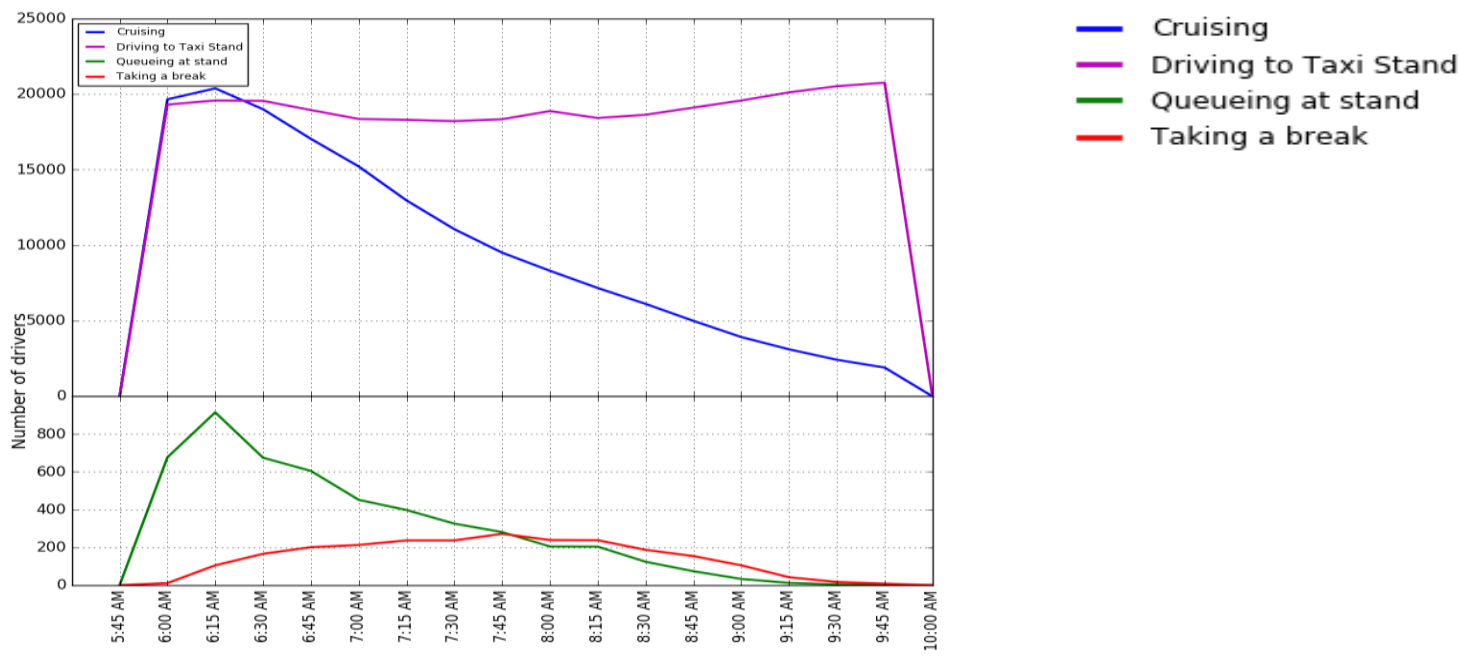

1 Figure 7: MoD driver behavior during simulation 

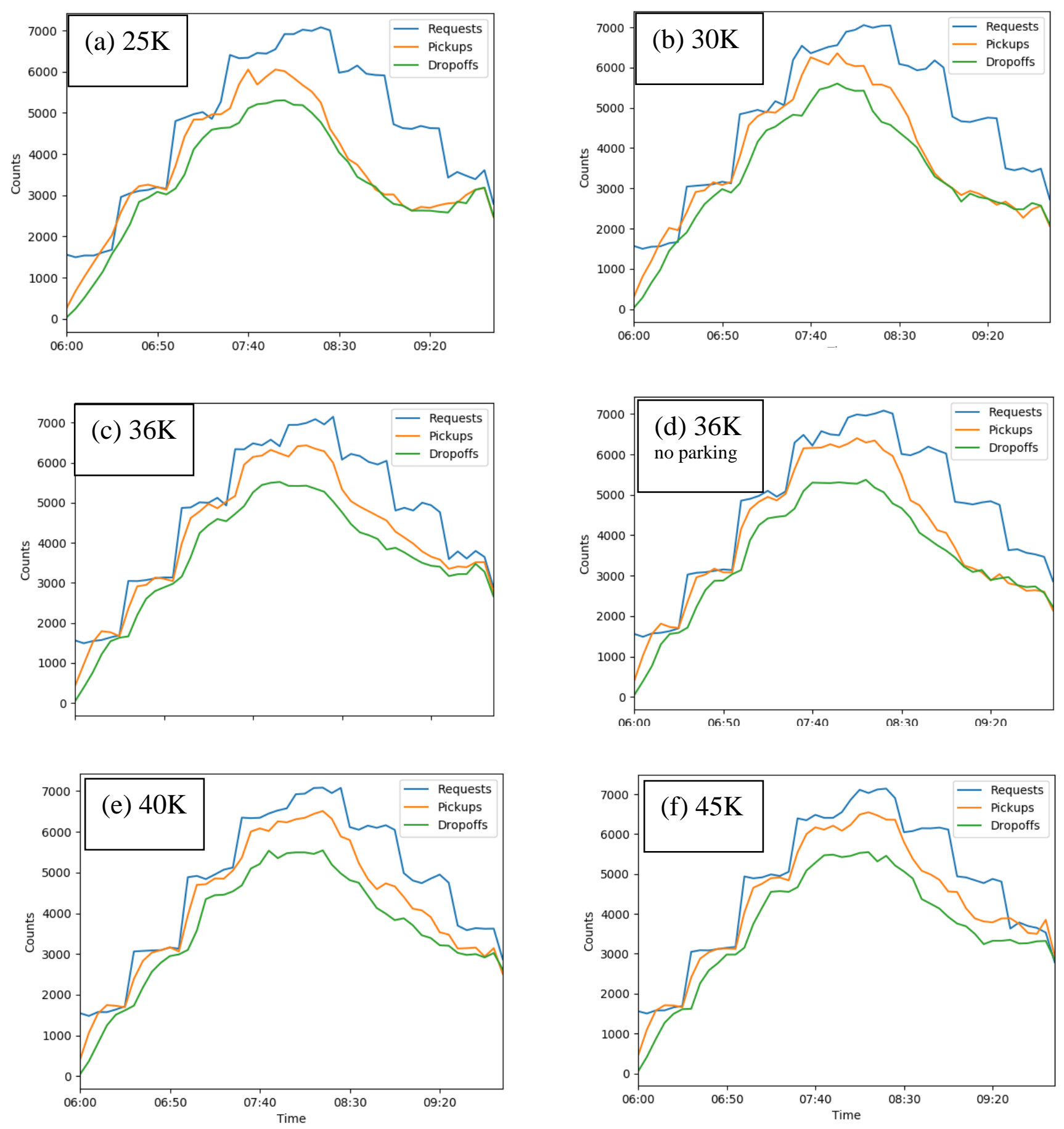

Figure 8: Controller performance for AMOD service under various fleet size scenarios, all implemented with parking except $(d)$. 

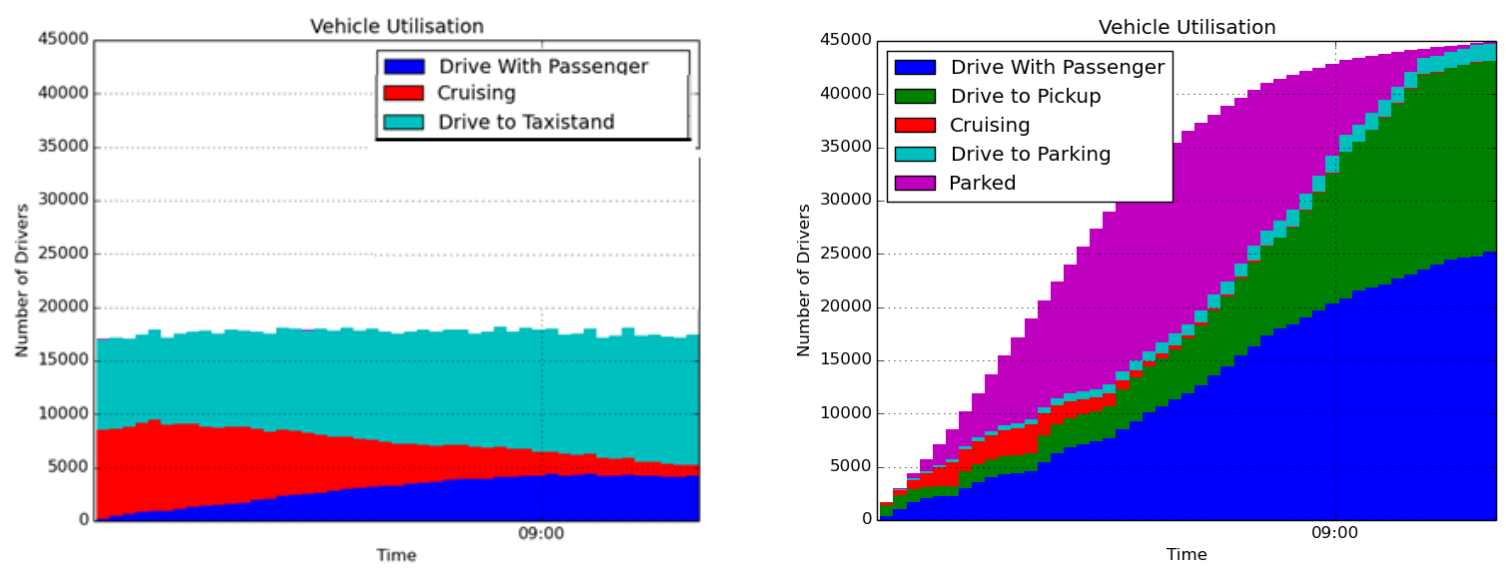

Figure 9: Vehicle utilization profiles for "Traditional MoD only" (20,000 vehicles) and best-case "AMoD only" (45,000 vehicles) scenarios 


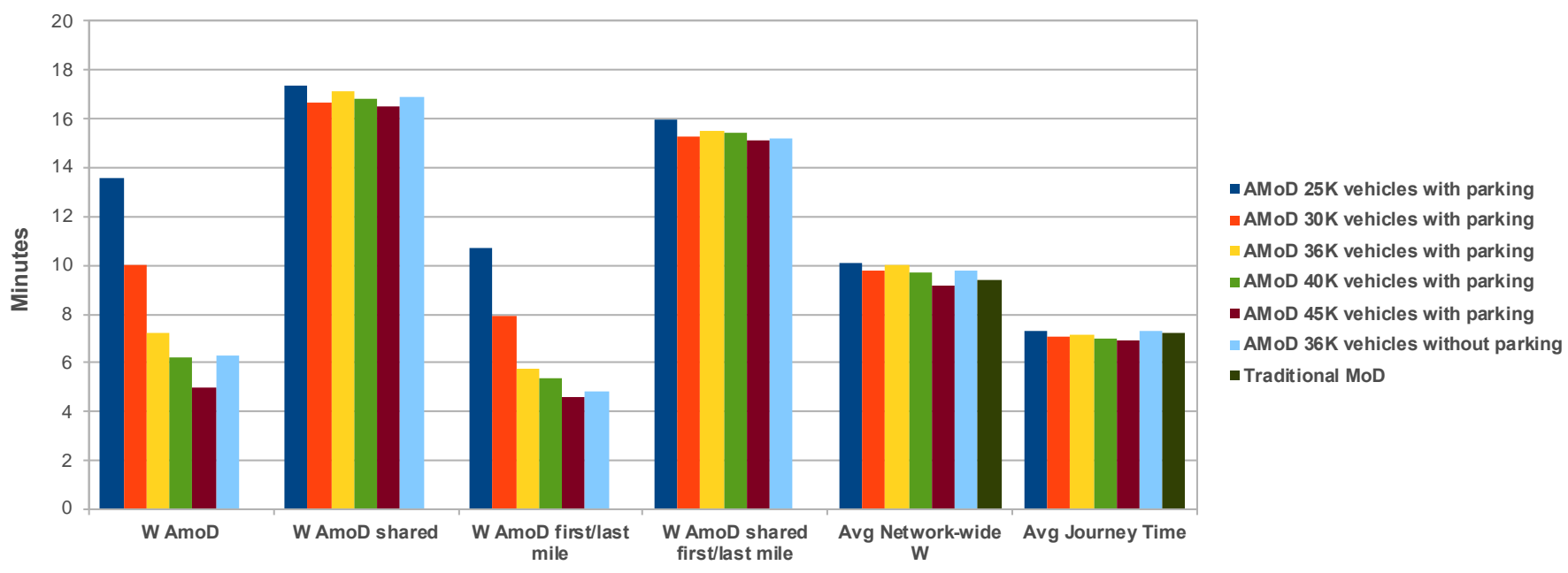

Figure 10: Waiting time $(W)$ across automated on-demand modes for the different scenarios (in minutes). Avg Network-Wide $W$ is the average $W$ over buses, trains and MoD. Journey Time captures average travel time across all modes. 
1 Table 2: Average travel times and distances for passenger-vehicle trips in both scenarios

\begin{tabular}{|c|c|c|c|c|c|c|c|c|}
\hline Scenario & $\begin{array}{l}\text { MoD Avg. } \\
\text { Travel Time } \\
\text { (min) }\end{array}$ & $\begin{array}{l}\text { MoD Avg. } \\
\text { Trip } \\
\text { Distance } \\
\text { (km) }\end{array}$ & $\begin{array}{l}\text { AMoD } \\
\text { Avg. } \\
\text { Travel } \\
\text { Time } \\
\text { (min) }\end{array}$ & $\begin{array}{l}\text { AMOD } \\
\text { Avg. Trip } \\
\text { Distance } \\
(\mathbf{k m})\end{array}$ & $\begin{array}{l}\text { AMoD } \\
\text { Pool } \\
\text { Avg. } \\
\text { Travel } \\
\text { Time } \\
\text { (min) }\end{array}$ & $\begin{array}{l}\text { AMoD } \\
\text { Pool } \\
\text { Avg. } \\
\text { Trip } \\
\text { Distance } \\
\text { (km) }\end{array}$ & $\begin{array}{l}\text { Car Avg. } \\
\text { Travel } \\
\text { Time } \\
\text { (min) }\end{array}$ & $\begin{array}{l}\text { Car Avg. } \\
\text { Travel } \\
\text { Distance } \\
(\mathbf{k m})\end{array}$ \\
\hline $\begin{array}{l}\text { Traditional } \\
\text { MoD }\end{array}$ & 14.5 & 10.1 & - & - & - & - & 14.6 & 9.4 \\
\hline AMoD & & & 13.2 & 8.0 & 20.3 & 13.7 & 15.6 & 10.0 \\
\hline
\end{tabular}

